\title{
LA PARTICIPACIÓN EN LAS COMUNIDADES RURALES: ABRIENDO ESPACIOS PARA LA PARTICIPACIÓN DESDE LA ESCUELA
}

\author{
Nancy Torres Victoria ${ }^{1}$ \\ Docente en la División de Educación Rural CIDE, Universidad Nacional \\ Heredia, Costa Rica
}

Recibido 18 de junio 2007 • Aprobado 15 de octubre 2007

\begin{abstract}
Resumen: El artículo se centra en el tema de la participación en la escuela rural, transita antes por el marco jurídico internacional y nacional del concepto y plantea interrogantes que tendrá que hacerse el docente rural que quiera abrir espacios de participación en la escuela rural. Además, incluye y desarrolla el concepto de capital social relacionándolo con el tema educativo.
\end{abstract}

Palabras clave: Participación, educación, legislación internacional.

\begin{abstract}
The article id based on the participation in the country school, It goes over the national and international legislation, is important to stand out the fact that this article establish questions that the personal teaching will affront if he wants to open a space to participate in country school. Besides it includes and develops the share capital concept and relate it whit the educational theme.
\end{abstract}

Key words: Participation, education, international legislation.

La participación busca influir en la toma de decisiones y lograr el cambio. La participación de la que hablamos, es la participación informada y voluntaria de todas las voces tradicionalmente silenciadas; la de las mujeres, la de los jóvenes, adolescentes, niños y niñas incluyendo, a todas aquellas personas que tienen diferentes capacidades y a aquellos grupos que están en situación de riesgo, en cualquier asunto que les concierne directa o indirectamente. La participación debe ser un valor que abarque todos los programas y se produzca en todos los escenarios desde el hogar, la comunidad, el centro educativo, al gobierno, desde el nivel local hasta el internacional.

La educación rural impulsa, como uno de sus principios fundamentales, la promoción de la participación de todos los escenarios y grupos de actores organizados en las comunidades. Sin

\footnotetext{
Historiadora y Socióloga, educadora. Actualmente se desempeña como profesora en la División de Educación Rural (DER) impartiendo cursos como Investigación-Acción, Investigación Lúdica, Diseño y Formulación de Proyectos en zonas rurales, Derechos Humanos de la Niñez, Educación no Formal, entre otros. Participa del equipo de investigación de la DER y tiene amplia experiencia trabajo en el campo de los derechos humanos, el derecho a la educación en procesos participativos comunitarios, en educación formal y no formal, con diferentes grupos etáreos, especialmente con grupos en situación de vulnerabilidad social. Correo electrónico: nancytatv@gmail.com
} 
embargo, las experiencias de participación de las comunidades son aún limitadas en cantidad y calidad; aunque, existe, cada vez más, un marcado interés por promoverlas.

En esta línea se realizan estudios, sistematizaciones, evaluaciones y se implementan proyectos para fortalecer a los diferentes actores sociales relacionados con la participación en las comunidades rurales, lo que constituye un aporte al proceso mismo. Por otro lado, también se evidencia un importante vacío en cuanto a metodologías de análisis, indicadores e instrumentos que permitan medir el desarrollo y orientación de la participación.

La participación, como derecho, está regulada desde 1948 en la Declaración Universal de los Derechos Humanos, artículo 19, que dice: "Todo individuo tiene derecho a la libertad de opinión y de expresión; este derecho incluye el de no ser molestado a causa de sus opiniones, el de investigar y recibir informaciones y opiniones, y el de difundirlas, sin limitación de fronteras, por cualquier medio de expresión”. Y en el articulo 20, que estipula: “...Toda persona tiene derecho a la libertad de reunión y de asociación pacíficas. ...Nadie podrá ser obligado a pertenecer a una asociación” (Organización de las Naciones Unidas [ONU], 1948, s. p.).

Así, la participación se constituye en uno de los cuatro principios rectores de los derechos humanos, que son: Indivisibilidad, universalidad, responsabilidad (rendición de cuentas) y participación. Es decir, que toda persona sin distingo de raza, estado civil, género, credo religioso, grupo etáreo, nacionalidad, pertenencia a grupo, entre otros, tiene derecho a participar activamente en la vida social, política, económica y cultural del país, y en la vida social, cultural y educativa de la escuela donde estudian sus hijas o hijos.

La participación desde el enfoque de derechos, se debe concebir como el reconocimiento del derecho que tiene toda persona a participar activamente, de manera informada, en todos aquellos asuntos, cuestiones, temas, actividades etcétera, que lo afecten, le interesen, le atañan.

El estudioso del tema, Crowley, señala que la participación es un principio directivo clave, un derecho facilitador, es decir que su cumplimiento contribuye a asegurar el cumplimiento de todos los demás derechos. Es un fin en sí mismo, no es simplemente un medio para lograr un fin, ni tampoco simplemente un proceso, es un derecho civil y político básico de todas las personas (Crowley, 1998).

El acceso a la información es una condición de primera base para el ejercicio de la participación. Para estar en condición de participar, se requiere tener acceso a la información relevante, presentada de manera comprensible, en un lenguaje accesible y sin códigos que disfracen o dificulten su comprensión.

La participación es una necesidad sentida desde los diferentes espacios entre los que la educación rural está comprometida. Desde la educación rural la participación se piensa concreta y real, pero, ¿cómo hacer para que la participación de la que hablamos no sea decorativa, utilitaria, parcial, simbólica? ¿Qué hacer para que todo aquello que pueda ir contra el derecho de las personas de la comunidad educativa a participar en los procesos de amplio espectro de la escuela, se erradique y se promueva una real y respetuosa participación? ¿Será que si contáramos con indicadores claros y precisos que señalen pistas y derroteros, podríamos abrir nuevas vías a la participación de la que tanto y con tanta vehemencia hablamos? ¿Qué es lo que esperamos que vengan a hacer las personas de la comunidad a la escuela rural? ¿Cómo trascender la participación en ferias, festivales y actividades de búsqueda de fondos para una acción puntual? ¿Qué condiciones debiéramos modificar para permitir que esto suceda? ¿Cuáles son las infraestructuras que más resistencia presentan a la participación y porqué? ¿Cuáles intereses se ven amenazados cuando se promueve la participación? ¿Cómo evitar que esto suceda? ¿Cuál es la información pertinente que debieran tener las personas que queremos hacer participar? 
A simple vista, se nota que las interrogantes son muchas. El tema no es simple. Sin embargo, la estructura, y no sólo la estructura física, pareciera diseñada para impedir la participación.

Al respecto, Durston (1997) plantea muy acertadamente que el paradigma emergente del capital social les sugiere a muchos investigadores que los programas de superación para la pobreza rural y para el fortalecimiento de la sociedad civil rural tendrían mejores resultados si tomaran en consideración los sistemas socioculturales presentes en las comunidades. Esta propuesta se basa en la idea, bastante difundida ya, de que las relaciones económicas están inmersas o incrustadas "embeddedness" (Granovetter, 1985, citado en Galán y Castro, 2004, p. 110) en el tejido social, lo cual es determinante para las formas y los resultados de las actividades económicas.

La teoría del capital social ofrece un marco especialmente adecuado para estudiar la sociedad rural, porque la reciprocidad interpersonal y la cooperación comunitaria son dos recursos tradicionales de la cultura rural y a la vez, elementos básicos del capital social.

La definición de capital social mantiene su base en la gestión y explotación de los recursos naturales por parte de un grupo de personas que comparten un espacio físico y geográfico, construyendo en este proceso sentido de pertenencia a una comunidad local, en que, las redes de relaciones interpersonales son esenciales no sólo para las estrategias económicas de los hogares y sus miembros, sino también para otros ámbitos cruciales de la vida humana, como la amistad, la religión, el esparcimiento y el sentido de pertinencia.

Esto nos lleva al segundo factor esencial en esta definición ampliada de capital social: los integrantes de una comunidad rural comparten un sistema sociocultural propio, en que las creencias y normas complementan las relaciones e instituciones sociales, y viceversa (Durston, 1997).

En síntesis, se podría afirmar que el capital social se construye y se conforma en grupos humanos que comparten formas de concebir, de relacionarse y de entender el mundo: su mundo.

Según el mismo autor, otro rasgo decisivo que suele dejarse de lado en la definición de capital social rural, es su inserción en el sistema social microrregional y nacional. En casi todos los países, el campesino ocupa uno de los últimos renglones de la escala social, y es objeto de explotación económica y exclusión social y política por parte de los grupos de mayor poder; fenómenos que suelen ser más intensos cuando los campesinos pertenecen a etnias con una historia de dominación por parte de otras. Este elemento tampoco ha sido analizado adecuadamente en las discusiones sobre el potencial del capital social.

El paradigma del capital social puede hacer un aporte significativo a la construcción de una visión prospectiva y proactiva del desarrollo social rural, porque se enmarca plenamente en el reconocimiento de lo local, y se basa en la detección y valoración de las fortalezas y capacidades propias de los grupos, las organizaciones locales. Por ende, la tarea de la escuela rural y del docente rural es no sólo analizar las formas propias de capital social y cultural contenidas en los sistemas socioculturales de las comunidades rurales, sino también entender qué sucede en la zona de interfaz donde este sistema se encuentra con el de los organismos estatales y propiciar los espacios para que, en los diferentes escenarios y los diferentes grupos comunitarios, puedan hacer su aporte, puedan dialogar y ofrecer su saber a la escuela.

La relación entre la participación de la comunidad en la gestión de la escuela rural y los logros en la equidad educativa, puede que no sean inmediatamente visibles, pero nadie ha de ignorar que cualquier intervención, proyecto o programa, funciona mejor si desde su diseño y formulación se ha contado con la participación de las personas que están directamente involucradas para la toma de las decisiones importantes del proyecto; es decir, si se ha tomado en cuenta y se ha involucrado el capital social y cultural local. 
Los habitantes de las comunidades rurales no son recipientes vacíos, beneficiarios como suelen llamarlos desde afuera, asignándoles un rol pasivo en la planificación. Por el contrario, los conocimientos del medio y de la compleja evolución del tejido socioeconómico local, superan con creces los conocimientos académicos o técnicos que pueden tener los llamados expertos sobre estos aspectos esenciales (Durston, 1997).

Este tejido socioeconómico, que algunos autores denominan el capital social, y en otros casos, el capital cultural de las comunidades, abarca las instituciones informales de la comunidad rural, y es también una red de intercambios de bienes y de información que resulta de vital importancia para promover participación.

El abrir espacios para la participación de los saberes instalados en la comunidad en la gestión de la escuela rural, es especialmente relevante para el logro eficiente y eficaz de mejoramientos en el aprendizaje de niñas y niños de escasos recursos económicos.

La escuela rural, y por ende, el docente rural, puede y debe establecer códigos de comunicación intercultural con la comunidad a la cual pertenece. En primer lugar, habría que conocer, respetuosamente, los procesos históricos, sociales, económicos, culturales que han tenido lugar en la comunidad, indagar sobre su origen, evolución, estado actual, desarrollo, desenlace, o cierre, siendo muy cuidadoso en este proceso de indagación, intentando incluir las perspectivas diferentes que sobre un mismo acontecimiento pueden existir, haciendo lo posible por no tomar partido.

En la medida en que padres y madres de familia puedan jugar roles más protagónicos en la gestión de la escuela, los logros pedagógicos y administrativos darán resultados visibles ya que esta es una forma simple de multiplicar las fuerzas dinamizadoras y ampliar los espacios para la acción.

No obstante, que se abran los espacios para la participación, no depende de los padres y las madres, depende -en las comunidades y escuelas rurales- únicamente del docente rural. Se requiere, entonces, de un docente rural que sea capaz de entender los códigos culturales, el lenguaje local y traducir la propuesta pedagógica tradicional -y no sólo el currículo-, adecuándolas a los procesos comunitarios locales; como quien dice, el docente rural será un docente bilingüe, que conoce perfectamente los códigos de la cultura amplia general, en los cuales está planteado el currículo, y es capaz de articularlo con los códigos culturales locales, sin importar su propia procedencia, ya que el conocimiento para realizar este empalme es el que ha debido adquirir en las aulas universitarias.

Un primer paso, es partir de que las comunidades no son un conjunto de carencias y obstáculos sin remedio. Como lo menciona Durston (1997), a muchos docentes les puede resultar extraña la idea de que la cultura de estas comunidades tiene que ser la base del aprendizaje, y de que estos adultos -en ocasiones analfabetas- tienen algo que aportar al proceso educativo.

En segundo lugar, hay que rechazar absolutamente la sugerencia de que el mejoramiento de los resultados de la educación rural requiere una transferencia parcial de poder a las madres y padres y su incorporación a la toma de decisiones en la escuela.

No, no se trata de hacer pedagogos a los padres y madres, ellas y ellos son participantes, el especialista debe ser el docente, pues como se anotaba anteriormente, es él quien está formado académicamente para jugar ese rol; en él se ha depositado la responsabilidad social para administrar y desarrollar el currículo, sólo que si lo hace abriendo espacios, seguramente su labor tendrá resultados de mayor calidad. Abrirse a la participación implica ceder poder, o si se quiere entender de otra manera, compartirlo. Este tipo de decisiones trae riesgos y ventajas. Sin duda alguna, las ventajas son más tangibles y mayores que los riesgos. Hace falta entonces, decisión y la firme convicción de que juntos somos más fuertes, más capaces, más eficientes. 


\section{REFERENCIAS}

Crowley, P. (1998). Participación infantil: Para una definición del marco conceptual. En B. Abegglen y R. Benes (Comp.), La participación de niños, niñas y adolescentes en el contexto de la Convención de los Derechos del Niño. Visiones y Perspectivas (pp. 9-16). Florencia, Italia: UNICEF.

Durston, J. (1997). La participación comunitaria en la gestión de la escuela rural. En E. Cohen (Aut.), Educación, eficiencia y equidad (pp. 68-84). Santiago, Chile: Ediciones SUR. Consultado el 22 de noviembre de 2007 de http://www.sitiosur.cl/r.php?id=714

Galán, J. L. y Castro, I. (2004). Las relaciones interorganizativas como fuente de capital social. Universia Business [Actualidad Económica], pp. 104-117. Consultado el 20, octubre, de 2007 de http://www.google.co.cr/search?hl=es\&q=Granovetter\%2B1985\%2Btejido+social\&btnG= Buscar\&meta=

Organización de las Naciones Unidas [ONU]. (1948). Declaración Universal de los Derechos Humanos. Consultado el 22, noviembre de 2007 de www.un.org/spanish/hr/ 